\title{
Malignant mesothelioma of tunica vaginalis: an extremely rare case presenting without risk factors
}

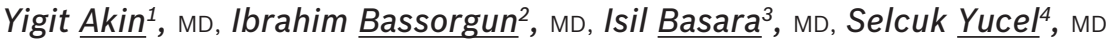

\begin{abstract}
Testicular tumours have many different manifestations, including hydrocele formation. Herein, we present an extremely rare case of testicular mesothelioma presenting with left hydrocele, but without risk factors. Left radical inguinal orchidectomy was performed, and pathological examination revealed a malignant mesothelioma of the tunica vaginalis of the testis. No infiltration of the spermatic cord was evident, and upon advanced radiological evaluation, no sign of metastasis was detected. Follow-up was still ongoing in our urology outpatient clinic at the time of this report. Although hydrocele is a simple and common condition that is easy to diagnose, a detailed investigation should be performed. Thus, when encountering a patient with hydrocele, the clinician should evaluate the possibility of the presence of an underlying testicular/paratesticular tumour, including a rare one such as mesothelioma of the tunica vaginalis.
\end{abstract}

Keywords: hydrocele, malignant mesothelioma, orchidectomy, testicular tumour

\section{INTRODUCTION}

Hydrocele is an abnormal water level in the scrotum, and occurs in approximately $1 \%$ of men over the age of 40 years. ${ }^{(1)}$ It is regarded as a benign condition and can be easily treated surgically. However, acquired hydrocele is usually idiopathic and can be triggered by trauma, infectious disease, or in rare instances, a testicular tumour. Physical examination and radiological evaluation can be of assistance in its diagnosis. In addition, the levels of markers of testicular tumours, such as alpha-fetoprotein $(\alpha \mathrm{FP})$, beta-human chorionic gonadotropin $(\beta-\mathrm{HCG})$, and lactate dehydrogenase (LDH), should be measured in the laboratory.

Malignant mesothelioma of the tunica vaginalis is an extremely rare cause of hydrocele. (1) Exposure to asbestos is a risk factor for mesothelioma, but the disease may also occur in the absence of any obvious risk factors. ${ }^{(2)}$ Malignant mesothelioma is usually fatal. Herein, we present an extremely rare case of a left testicular hydrocele, which was subsequently diagnosed as testicular mesothelioma. Radical inguinal orchidectomy was performed. Although our patient did not exhibit any risk factor for malignant mesothelioma, pathological examination revealed malignant mesothelioma of the tunica vaginalis.

\section{CASE REPORT}

A 49-year-old man with a left testicular mass was referred to our urology outpatient clinic. The mass had appeared seven months earlier without any history of trauma. On physical examination, a hard and painless mass was evident in the left scrotum and was suggestive of a hydrocele on palpation. No left inguinal hernia was evident. The right testis was normal, as was the scrotal skin. No palpable lymph node was detected in the pelvic or inguinal areas. Ultrasonography, performed in our radiology unit, revealed an increase in the size of the left scrotum, with many multiloculated cysts of different sizes. The parenchyma and size of the left testis were normal (Fig. 1). The levels of $\alpha$ FP, $\beta$-HCG, and LDH were not elevated, and chest radiography was normal. Computed tomography (CT) of the abdomen, pelvis and chest showed no sign of metastasis to the lung or any pathological lymph node in the retroperitoneum. Although the levels of tumour markers were normal, the rapid growth of the mass and the presence of multiloculated cysts encouraged us to perform left radical inguinal orchidectomy.

Microscopically, the tumour contained papillary structures, with confluent sheets and nests of polygonal tumour cells, and large necrotic areas (Fig. 2). The tumour cells had abundant eosinophilic cytoplasm, coarse chromatin, and prominent nucleoli. There was also frequent mitosis. Immunohistochemical tests were performed to help elucidate the nature of the tumour. Diffuse immunoreactivity was evident against mesothelial markers including calretinin, cytokeratin 5/6, and Wilms' tumour gene 1 (Fig. 2d), whereas the tissue was negative for both placental alkaline phosphatase and alpha-inhibin. The pathology report indicated malignant mesothelioma of the left tunica vaginalis without infiltration of the spermatic cord.

We consulted with medical oncologists, who suggested that no additional treatment was required. Therefore, the patient had been on follow-up in our outpatient clinic for four years and continued to do so at the time of this report.

\section{DISCUSSION}

A mesothelial tumour can arise from any tissue that has a mesothelial membrane. Testicular mesothelioma, a testis-specific tumour, is an aggressive form of cancer, ${ }^{(1)}$ developing from the mesothelium and covering the tunica vaginalis, epididymis, spermatic cord, or tunica albuginea. ${ }^{(2)}$

Testicular mesothelioma can occur in male patients of varying age, but is most common in those aged between 55 and 75 years. ${ }^{(3)}$ Although the developmental mechanism of testicular mesothelioma remains poorly known, exposure to asbestos is

${ }^{1}$ Department of Urology, Harran University School of Medicine, Sanliurfa, ${ }^{2}$ Department of Pathology, Akdeniz University School of Medicine, Antalya, ${ }^{3}$ Department of Radiology, Harput State Hospital, Elazig, ${ }^{4}$ Department of Urology, Akdeniz University School of Medicine, Antalya, Turkey

Correspondence: Dr Yigit Akin, Assistant Professor, Department of Urology, Harran University School of Medicine, Sanliurfa 63100, Turkey. yigitakin@yahoo.com 

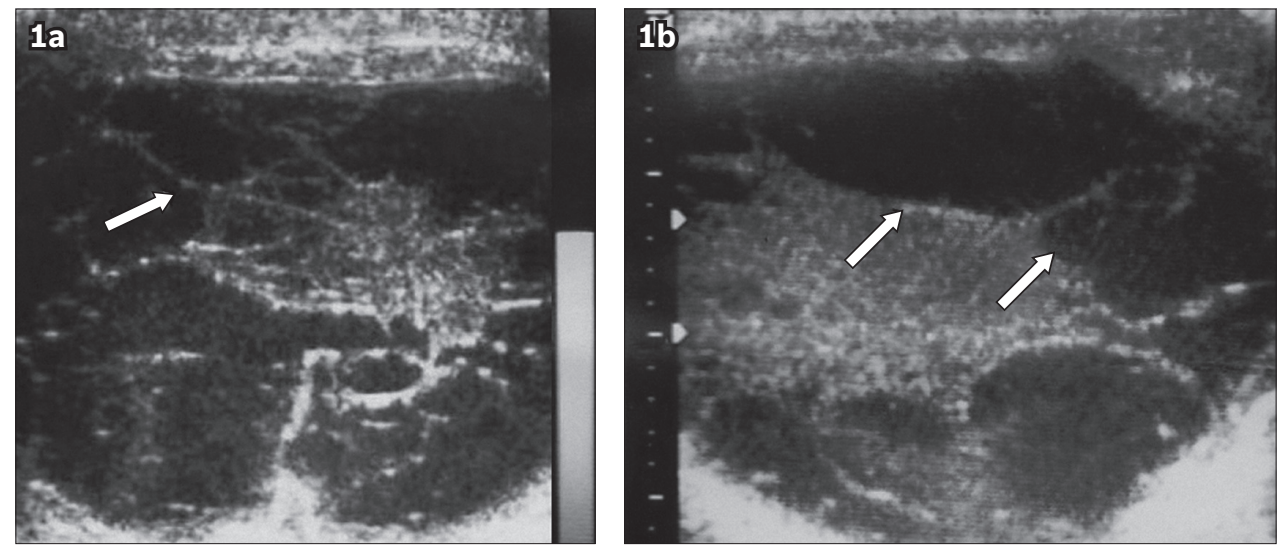

Fig. 1 (a-b) Scrotal ultrasonographs show an increase in the size of the left scrotum, which was filled with many multiloculated cysts of different sizes (arrows). The cysts lay within the paratesticular region (tunica vaginalis). The parenchyma and size of the left testis were normal.
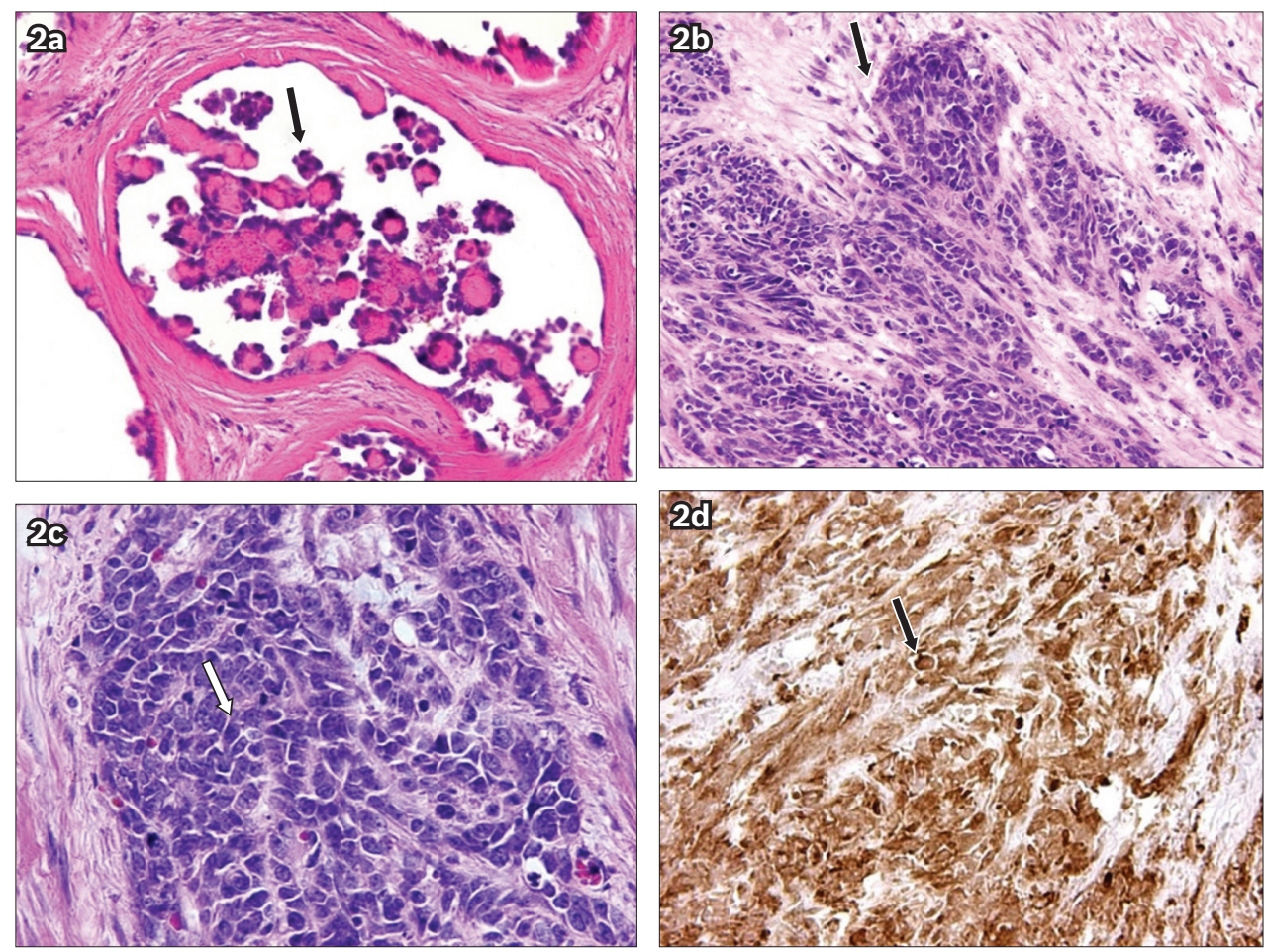

Fig. 2 Photomicrographs of the tumour cells show (a) papillary structures (arrow) (Haematoxylin \& eosin $\times 400$ ), (b-c) confluent sheets and nests of polygonal tumour cells (arrows) (Haematoxylin \& eosin, $\times 200$ and $\times 400$, respectively), and (d) intense WT-1 positivity (arrow) (Haematoxylin \& eosin, $\times 100$ ).

a well-known risk factor, as are trauma, long-term hydrocele, and herniorrhaphy. ${ }^{(4-6)}$ In 2006, Hatzinger et al noted that more than 80 cases of testicular mesothelioma had been reported in the literature. ${ }^{(7)}$ To these, we add our present case of testicular mesothelioma in a patient aged under 55 years, exhibiting no risk factor and with no known history of asbestos exposure (either occupational or proximal).

Patients with testicular mesothelioma may present with scrotal problems, as in our case. ${ }^{(3)}$ The rarity of testicular mesothelioma poses great challenges in diagnosis. ${ }^{(8)}$ Physical examination and radiography are used to detect lesions. Ultrasonography is noninvasive and simple, and $90 \%$ accurate when used to detect testicular tumours. ${ }^{(9)}$ Furthermore, it can be used for differential diagnosis to exclude: (a) epididymitis; (b) florid mesothelial hyperplasia; (c) adenomatoid tumour; (d) carcinoma of the rete testis; (e) serous papillary tumour; ( $f$ ) various types of testicular germ cell tumours including seminomas, embryonal carcinomas, and intratubular germ cell tumours; (g) and pleomorphic sarcomas, ${ }^{(10)}$ all of which should be considered differential diagnoses. The levels of testis tumour markers including $\alpha F P$, $\beta-H C G$, and LDH can aid in the diagnosis of testicular tumours. In our case, however, the levels of the markers were normal.

Seminomas are the most common testicular tumours in patients aged less than 50 years. The levels of testicular tumour markers may be normal in such patients, as in our case, and ultrasonography may allow an intraparenchymal mass to be diagnosed in seminoma patients. ${ }^{(9)}$ In our case, the left scrotum was increased in size and many multiloculated cysts of different sizes were evident, but the parenchyma and size of the left testis were normal. The ultrasonography findings and patient 
history suggested an uncommon malignant tumour of the testis. Under such circumstances, CT should be performed to stage the tumour, diagnose distant metastases, and especially evaluate the pathological status of the retroperitoneal lymph nodes. ${ }^{(11)}$ We found no distant metastasis in our case. Apart from clinical evaluation, pathological findings are important in terms of differential diagnosis. Immunohistochemical staining was performed to aid in accurate diagnosis. Diffuse immunoreactivity for mesothelial markers, including calretinin, cytokeratin 5/6, and Wilms' tumour gene 1, was evident. On the other hand, staining for placental alkaline phosphatase (a marker of seminomas) and alpha-inhibin (a marker of sex cord stromal tumours) was negative.

Treatment of testicular mesothelioma may feature (sequential) surgery, radiation therapy and chemotherapy. Combined treatment options may be required for patients in the advanced stage. ${ }^{(12)}$ If the disease is localised, as in our case, radical inguinal orchidectomy is the optimal primary surgical approach. If any sign of metastasis is evident in the inguinal lymph nodes, inguinal lymph node dissection is required. The utility of adjuvant chemotherapy and/or radiotherapy has not been clearly shown because of the limited number of reported cases. Cisplatin and pemetrexed can be used as chemotherapies for testicular mesothelioma, ${ }^{(13)}$ while radiotherapy may be helpful in preventing disease recurrence. ${ }^{(7)}$ Our patient did not receive radiotherapy because he was surgically treated at an early stage and showed no sign of metastasis.

The mortality rate from testicular mesothelioma has been reported to be $53 \%$ over a mean follow-up time of two years. ${ }^{(6)}$ Follow-up of our patient has been ongoing for four years in our outpatient clinic, and will continue for the period considered appropriate by our medical oncologists.

Our case differs from those in the literature in that we did not identify any risk factors for testicular mesothelioma (such as asbestos exposure, trauma, or long-term hydrocele), and our patient was young, with an early-stage disease. Triggers of testicular mesothelioma are still under study. In a recent work, it was argued that simian virus 40 (SV40) was a cause of mesothelioma. ${ }^{(14)}$ Moreover, the delayed effect of asbestos exposure and the long latency of the tumour are also significant causes. ${ }^{(15)}$ Thus, the testicular mesothelioma of our case may be associated with SV40 infection, a delayed effect of asbestos exposure, or an unknown cause.

In conclusion, a hydrocele should be closely monitored. Testicular mesothelioma is extremely rare but should be kept in mind when diagnosing patients with a testicular mass, even if they have no history of exposure to a risk factor.

\section{REFERENCES}

1. Park YJ, Kong HJ, Jang HC, et al. Malignant mesothelioma of the spermatic cord. Korean J Urol 2011; 52:225-9.

2. Perez-Ordonez B, Srigley JR. Mesothelial lesions of the paratesticular region. Semin Diagn Pathol 2000; 17:294-306.

3. Plas E, Riedl CR, Pflüger H. Malignant mesothelioma of the tunica vaginalis: review of the literature and assessment of prognostic parameters. Cancer 1998; 83:2437-46.

4. Jones MA, Young RH, Scully RE. Malignant mesothelioma of the tunica vaginalis. A clinocopathologic analysis of 11 cases with review of the literature. Am J Surg Pathol 1995; 19:815-25

5. Attanoos RL, Gibbs AR. Primary malignant gonadal mesotheliomas and asbestos. Histopathology 2000; 37:150-9.

6. Gürdal M, Erol A. Malignant mesothelioma of tunica vaginalis testis associated with long-lasting hydrocele: could hydrocele be an etiological factor? Int Urol Nephrol 2001; 32:687-9.

7. Hatzinger M, Hacker A, Langbein S, Grobholz R, Alken P. Malignant mesothelioma of the testes. Aktuelle Urol 2006; 37:281-3.

8. Mak CW, Cheng TC, Chuang SS, et al. Malignant mesothelioma of the tunica vaginalis testis. Br J Radiol 2004; 77:780-1.

9. Blaivas M, Brannam L. Testicular ultrasound. Emerg Med Clin North Am 2004; $22: 723-48$

10. Yen $\mathrm{CH}$, Lee CT, Su CJ, Lo HC. Malignant mesothelioma of the tunica vaginalis testis: a malignancy associated with recurrent epididymitis? World J Surg Oncol 2012; 10:238.

11. De Klerk NH, Musk AW. Epidemiology of Mesothelioma. In: Robinson BWS, Chahinian AP, eds. Mesothelioma. London: Martin Dunitz Ltd, 2002: 339-49.

12. Yan TD, Welch L, Black D, Sugarbaker PH. A systemic review on the efficacy of cytoreductive surgery combined with perioperative intraperitoneal chemotherapy for diffuse malignancy peritoneal mesothelioma. Ann Oncol 2007; 18:827-34.

13. Ceresoli GL, Zucali PA, Favaretto AG, et al. Phase II study of pemetrexed plus carboplatin in malignant pleural mesothelioma. J Clin Oncol 2006; 24:1443-8.

14. Jasani B, Gibbs A. Mesothelioma not associated with asbestos exposure. Arch Pathol Lab Med 2012; 136:262-7.

15. Goel A, Agrawal A, Gupta R, Hari S, Dey AB. Malignant mesothelioma of the tunica vaginalis of the testis without exposure to asbestos. Cases J $2008 ; 1: 310$. 